\title{
Adaptation to prism-displaced vision: The importance of target-pointing'
}

Two experiments investigated the hypothesis that the experience of manually pointing at visual targets enhances motoric adaptation to prism-displaced vision. Experiment 1 indicated that when adaptation was measured by means of redirected pointing behavior (negative aftereffect) it varied directly with the specificity of the target, the least adaptation occurring when no target was available. This relationship was not observed when adaptation was measured in terms of a shift in the felt position of the prism-exposed hand (proprioceptive shift). Experiment 2 demonstrated that after double the prism-exposure trials used in Experiment 1, target-pointing experience continued to enhance adaptation (as indexed by both types of adaptation measure). In both experiments negative aftereffect was significantly larger than proprioceptive shift for all experimental conditions and the two measures were not correlated. These latter two findings cast doubt on Harris's notion that negative aftereffect is entirely the result of altered position sense.

When the visual field is laterally displaced by a wedge prism, initial attempts to reach for objects are in error. The $S$, however, rapidly learns to compensate for the rearrangement and continues to make this correction after the prism is removed, hence once again missing the target. This error in the direction opposite to that of the visual displacement is generally referred to as the "negative aftereffect."

Motoric adaptation to rearranged vision has been the subject of investigation for over a century. In recent years a large number of studies which have carefully analyzed the adaptive process have resulted in disagreement concerning both the conditions necessary for its instigation and the nature of its end product. Held $(1961,1962,1964)$ has concluded that adaptation to prism-displaced vision results in a recorrelation of neural "efferent copies" with the rearranged movementproduced visual feedback ("reafference"). According to Held's theory, the organism must engage in active motor behavior if visuomotor adaptation is to occur (e.g., Held \& Hein, 1958; Held \& Schlank, 1959; Held \& Bossom, 1961; Held \& Mikaelian, 1964).

Harris (1963a, b, 1964, 1965), on the other hand, has argued that active exposure to visual rearrangement leads to a change in the felt position (or the felt direction of movement) of those body parts that have been seen during the adaptation period. In short, the body comes to feel as if it is located where it was seen through the distorting spectacles. Harris (1965), for example, showed that if $\mathrm{S}$ viewed his actively moving right hand through a prism and then was asked to close his eyes and point to his right index finger with his left (unexposed) hand, he would miss in the direction of the visual displacement.

Both Held and Harris agree that two conditions necessary for visuomotor adaptation are: (1) a stable visual rearrangement and (2) active motor involvement [Harris suggests the possibility that felt body position is especially malleable when the musculature is in an active state $(1965, \mathrm{p} .441)]$. There is a difference, however, in their views concerning the information that the organism must use in order to adapt. According to Held, this information arises from the discrepancy between the usual reafference contingent on a given motor movement and the new reafference that occurs because of the distorting spectacles. Harris is not as clear about the causes of adaptation as he is about its end product, but he suggests that the crucial information might be the discrepancy between seen and felt position of the body (Harris, 1965, p. 440).

In a preliminary study the present investigator elicited adaptation that could be attributed to neither Held's nor Harris's suggested sources of information. It was discovered that Ss who were instructed to compensate for their initial target-pointing errors manifested nearly twice the level of adaptation (i.e., negative aftereffect) achieved by Ss instructed to avoid correcting for their errors. ${ }^{3}$ Because both conditions entailed conflicts between old and new reafference and between. felt and seen body position, it appears that some third source of information was provided to the Ss who were allowed to correct their pointing responses. More generally, it may be hypothesized that there exist many usable sources of information for the adaptive process and that the more sources present in an experimental situation, the more rapid and complete the resulting adaptation. This general notion, which may be referred to as the "Information Hypothesis," has been suggested by Coren (1966), Howard and Templeton (1966), Rock (1966), and Wallach (e.g., 1963, 1968). It was the purpose of the present experiments to subject this hypothesis to a test by manipulating a relatively neglected source of information-the target-pointing error.

\section{EXPERIMENT}

The first experiment tested the prediction that visuomotor adaptation will be more complete if target-pointing experience is provided than if it is not. In addition, the specificity of the target was varied, the expectation being that the more apparent the target, the greater the enhancement of adaptation.

\section{Method}

Subjects. One-hundred students of both sexes from an introductory psychology course volunteered to be Ss in the present experiment. Their mean age was approximately 20 years and the majority were right-handed.

Apparatus. Figure 1 shows the apparatus used. An 11-deg displacement of the visual field to S's right was effected by a wedge prism mounted in front of the right eye piece of a pair of war surplus goggles. Two measures of adaptation were recorded: (1) the negative aftereffect (NA) and (2) shift in the felt position of the right index finger (the finger seen during exposure to the visual rearrangement). The latter measure will hereafter be referred to as "proprioceptive shift" (PS). The NA score was operationally defined as a shift in the average postexposure pointing localization to the left of the average preexposure localization. This shift represents a compensation for the rightward visual displacement induced by the prism. The PS score represented the difference between pre- and postexposure measures of where $S$ felt his unseen right index finger was located. The method by which these measures were taken will be described below. According to Harris, the shift in felt finger position should be in the direction of the visual displacement-in this instance, to S's right.

Measurement of $N A$. The procedure for measuring pre- and 


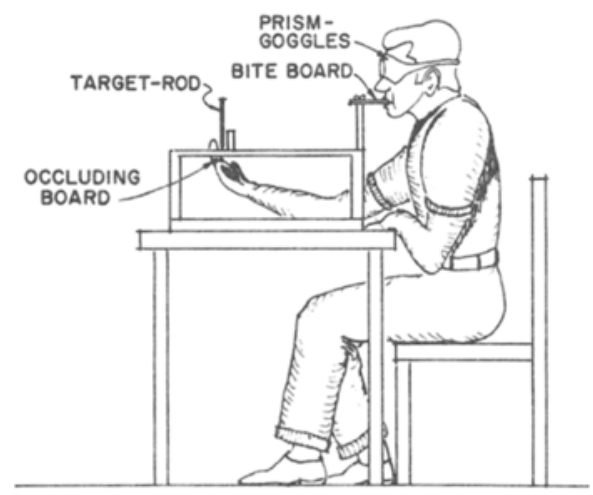

B

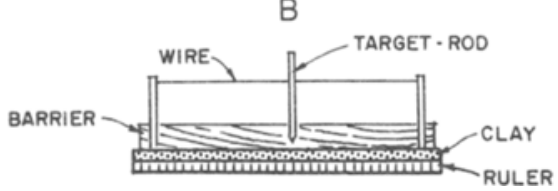

Fig. 1. (A) Side view of apparatus used to measure S's target-pointing accuracy and felt finger position; (B) front view of the same apparatus.

postexposure target-pointing accuracy was as follows: The $S$ sat in a chair, facing E (see Fig. la). Between them was situated a horizontal "occluding board," elevated 12 in. above the table, which kept $S$ from seeing his right pointing arm. The target at which $\mathrm{S}$ aimed was an 8-in. vertical rod, located $40 \mathrm{~cm}$ from his eyes, at the far edge of the occluding board. The $S$ was instructed to reach under the board and attempt to curl his right index finger around its far edge at a place directly opposite the rod. However, by means of a removeable barrier (see Fig. 1b), he was kept from seeing his finger when it emerged from behind the board and, hence, he was incapable of noting the accuracy of his responses. In order that a precise measure of S's target-pointing accuracy be made, E taped a thin wire to the ventral surface of S's right index finger. The wire deposited a mark in a strip of clay which was located on the upper half of the far edge of the occluding board (see Fig. 1b) and a ruler placed adjacent to the clay was used in measuring the error in localization. The $\mathrm{S}$ was required to make 15 preexposure and 15 postexposure attempts to align his finger with the rod, although (unknown to $S$ ) only the last 10 of each set of 15 responses were actually recorded. During pre- and postexposure trials $S$ wore prismless goggles with the left eye occluded.

Measurement of PS. When measuring felt finger position $\mathrm{S}$ was required to close his eyes and to allow his right index finger to be placed by $E$ in a hole that went half-way through the underside of the occluding board, near its far edge. The S was given a felt pen to hold in his left hand and was instructed to reach over the occluding board and to leave a mark on a strip of paper at a place that felt as if it were directly above his finger. The $S$ kept his eyes shut throughout this entire performance. He made 10 finger-pointing attempts before and 10 after wearing the prism. After each response $S$ brought his left hand back to the near edge of the board before reaching forward for his next attempt to point at the stationary and unseen right index finger. For practical reasons the postexposure measure of felt finger position occurred prior to the postexposure measure of target-pointing accuracy. This reversed the order of the preexposure measures.

Four prism-exposure conditions and one control (no prism) condition were used, with $20 \mathrm{Ss}$ in each group. Before donning the prism goggles, Ss in the experimental groups were informed of the wedge prism and its effects. During the prism-exposure period $S$ again reached under the occluding board. However, as a consequence of the removal of the wooden barrier, he was now allowed to see his right index finger as it curled around the far edge of the board. All groups were given 15 exposure trials. During the preexposure, exposure, and postexposure trials, and while felt finger position was being measured, S's head movements were restricted by means of a bite board. The groups differed from each other in regard to the task in which they engaged during the exposure period.

Target-Object (T-O) group. The $\mathrm{S}$ was instructed to immediately and completely compensate for his initial prism-induced error in pointing at the target. This group was expected to adapt to the greatest extent of any of the four prism-exposed groups, because $S$ was able to compensate for the visual displacement in reference to a very obvious target.

No-Target $(N-T)$ group. The rod was removed and $\mathrm{S}$ was instructed to bring his right index finger around the far edge of the board in the same manner as he would if the target were present. In addition, $S$ was requested to vary randomly from trial to trial the position in which he placed his finger. To further reduce the possibility of target-pointing experience $S$ was required to close his eyes while moving the finger along the underside of the board and to avoid opening them until just prior to curling his finger around its edge. This group was expected to adapt the least of the experimental groups.

Target-Image (T-I) group. The $\mathrm{S}$ was to point at the visually displaced image of the target and to avoid compensating for the prism-induced error. If $S$ succeeded in following these instructions, his finger would appear from behind the occluding board at a position about 3 in. to the right of the rod (the amount of the visual displacement). The T-I group was a replication of the "image-pointing" condition of the pilot study that prompted the present experiments. It was expected that this group would adapt less than the T-O group but more than the N-T group. It can be seen that $\mathrm{Ss}$ who were instructed to aim at the displaced image of the rod were placed in a conflict situation, i.e., they were being asked to aim at something and at the same time to continue to miss it. In the original pilot study some Ss spontaneously reported that in order to resolve this conflict they instructed themselves to point at an "imaginary target," a few inches to the right of the rod. If this activity were true for most or all the Ss, then this group was getting target-pointing experience, but the target was less concrete than that confronting the $T-O$ group. For this reason the amount of adaptation for the T-I group was expected to fall somewhere between that of the T-O and N-T groups.

Imaginary-Target (I-T) group. The $S$ was instructed to aim for a position $3 \mathrm{in}$. to the right of where he saw the rod through the prism. On the first trial $S$ could expect to miss this location by approximately the amount by which the prism displaced his vision (hence, bringing the finger around the edge of the board about $6 \mathrm{in}$. from the rod). On the subsequent trials, however, he was to compensate for the visual rearrangement, so that he would point accurately at the "imaginary target." The I-T group served as a test of the hypothesis that Ss in the T-I group would instruct themselves to point at a position to the right of the rod. With the assumption that this hypothesis was correct, it was predicted that there would be little or no difference between the adaptation levels of the T-I and I-T groups.

No-Prism (N-P) group. The $\mathrm{S}$ wore prismless goggles and was instructed to point directly at the rod. Naturally, zero adaptation was expected.

In summary, it was predicted that the $\mathrm{T}-\mathrm{O}$ group would 
Table 1

Mean (cm) Negative Aftereffect and Proprioceptive Shift: Experiment 1

\begin{tabular}{lcc}
\hline & \multicolumn{2}{c}{ Adaptation } \\
\cline { 2 - 3 } Group & NA & PS \\
\hline T-O & +4.79 & +2.65 \\
I-T & +3.79 & +1.87 \\
T-I & +3.27 & +1.51 \\
N-T & +2.37 & +1.90 \\
N-P & -.12 & +.08 \\
\hline
\end{tabular}

Note: + denotes deviation in the direction compensatory for the prism-displacement; - denotes deviation in the non-compensatory direction.

adapt the most, the I-T and T-I groups an intermediate (and equal) amount, and the N-T group least of all the experimental groups, although significantly more than the control group.

\section{Results and Discussion}

The results of Experiment 1 for both measures of adaptation are summarized in Table 1 . Using a one-tailed $t$ test, with $95 \mathrm{deg}$ of freedom, and the technique of planned orthogonal comparisons (Hays, 1963), it was determined that: (1) NA for the T-O group was significantly greater than the mean NA of the three remaining experimental groups $(t=3.05, p<.005) ;(2)$ the difference between the I-T and T-I groups failed to reach significance $(t=.79, p>.10) ;(3)$ mean NA for the latter two groups was significantly greater than that manifested by the $\mathrm{N}-\mathrm{T}$ group $(\mathrm{t}=2.02, \mathrm{p}<.025)$; and (4) mean NA of the four experimental groups was significantly greater than that of the control group $(t=6.98, p<.001)$. The findings were all as predicted.

When the same comparisons were made with regard to PS, the only significant differences were (1) between the T-O group and the other experimental groups $(t=1.71, p<.05)$ and (2) between the four experimental groups and the control group $(\mathrm{t}=3.80, \mathrm{p}<.001)$.

The mean NA-PS difference score for the experimental Ss was found to be significantly greater than zero $(t=5.41$, $\mathrm{df}=79, \mathrm{p}<.001)$. An examination of Table 1 indicates that NA was larger than PS for each of the experimental groups.

The relationship between $N A$ and target-specificity. It is apparent that in Experiment 1 prism exposure elicited both NA and PS. Only NA, however, clearly differentiated the experimental groups. Why this discrepancy in results as a function of dependent variable occurred is not certain at this time. If discussion is limited to the NA measure, the results of Experiment 1 confirm the hypotheses that target-pointing enhances visuomotor adaptation and that the more concrete the target, the greater the enhancement. A discussion of possible reasons why target-pointing facilitates NA will be delayed to a later section. It was found also that $S$ s who were instructed to point at the "imaginary target" did not significantly differ with regard to NA from Ss instructed to aim for the displaced image of the target. This finding supports the prediction that $S s$ in the latter group would instruct themselves to point at an "imaginary target."

The difference between NA and PS. An unexpected finding was that NA exceeded PS for all four experimental groups. This result poses a problem for Harris's theory of visuomotor adaptation. Adherents to this theory contend that the pointing aftereffect is a straightforward manifestation of altered proprioception. That is, by pointing to the left of the target, after the prism is removed, $\mathrm{S}$ is indicating that he feels $\mathrm{h}$ is arm is located to the right of its true position. According to this notion, the arm should feel displaced as far to the right as $S$ misses the target to the left when pointing with this arm. One possible explanation for the inequality of NA and PS found in the present experiment is that it was an artifact of the difference between the methods used to measure each "type" of adaptation. On the other hand, if NA and PS are one and the same, the correlation between them should approach +1.0 , even if, for methodological reasons, there is a systematic discrepancy in absolute size. However, a Pearson productmoment correlation between the two measures of adaptation for Ss in all four experimental groups produced an $\mathrm{r}$ of -.06 , which is not significantly greater than zero $(t=.53, \mathrm{df}=78$, $p>.25)$. Thus, the discrepancy in size between the mean NA and PS scores that was found in Experiment 1 may be indicative that the two responses are not, as Harris maintains, simply different ways of measuring a change in felt arm position. An alternative theory will be proposed in a later section.

\section{EXPERIMENT 2}

The results of Experiment 1 leave little doubt that pointing at either a concrete or an "imaginary" target produces greater adaptation (NA) than prism exposure in the absence of a target. However, since only 15 exposure trials were used, it could be maintained that the enhancement of adaptation as a result of target-pointing experience was only of a short-term nature. Experiment 2 attempted to discover whether or not adaptation would continue to be facilitated by target-pointing experience after twice the number of prism-exposure trials used in Experiment 1.

\section{Method}

Subjects. Sixteen Ss from the population used in Experiment 1 served as their own control in two experimental conditions.

Apparatus. The apparatus was identical to that used in Experiment 1. Both felt finger position and target-pointing accuracy were measured before and after prism exposure and in the same manner as in the first experiment.

No-Target $(N-T)$ condition. As in Experiment $1, \mathrm{~S}$ brought his finger around the far edge of the occluding board, without relating it to a target. The $S$ kept his eyes closed until just prior to bringing the finger into view and randomly varied from trial to trial the position in which he placed his finger.

Target-Object $(T-O)$ condition. The $\mathrm{S}$ was instructed to compensate for the visual displacement and to point correctly at the rod. A difference between this condition and the T-O group of Experiment 1 was that in the present experiment the position of the target was randomly shifted by $E$ from trial to trial.

The two experimental conditions were presented exactly one week apart and the order in which they occurred was counterbalanced across Ss. The major difference between the two experiments was that 30 prism-exposure trials were used in Experiment 2, in contrast to the 15 trials in Experiment 1.

\section{Results and Discussion}

The results are presented in Table 2. Because $t$ tests revealed no difference between the two orders of presentation, the data from both orders were combined for the subsequent analysis. One-tailed t tests of the difference scores disclosed that the T-O condition elicited significantly greater NA than did the Table 2

Mean (cm) Negative Aftereffect and Proprioceptive Shift: Experiment 2

\begin{tabular}{lcc}
\hline & \multicolumn{3}{c}{ Adaptation } \\
\cline { 2 - 3 } & NA & PS \\
\hline $\mathrm{T}-\mathrm{O}$ & +5.06 & +2.26 \\
$\mathrm{~N}-\mathrm{T}$ & +2.67 & +1.20 \\
\hline
\end{tabular}

Note: + denotes deviation in the direction compensatory for the prism-displacement. 
N-T condition $(t=3.85, \mathrm{df}=15, \mathrm{p}<.001)$ and greater PS $(t=1.80$, df $=15, p<.05)$. Combining the NA scores of each of the two experimental conditions and comparing them with the combined PS scores indicated that the difference in size favoring NA was significant $(t=4.04, \mathrm{df}=15, \mathrm{p}<.001)$. Finally, a Pearson product-moment correlation of the two measures was +.27 , which is not significantly greater than zero $(\mathrm{t}=1.05, \mathrm{df}=14, \mathrm{p}>.10)$.

The results of Experiment 2 indicate that target-pointing experience continues to enhance visuomotor adaptation after 30 prism-exposure trials. Contrary to the results of Experiment 1, this effect was apparent in PS as well as NA. It remains to be seen how much experience in a targetless situation would be necessary in order that Ss "catch up," with regard to adaptation, to Ss pointing at some form of visual target, although Held and Freedman (1963) found that $1 / 2 \mathrm{~h}$ of active motor movements without target-pointing led to complete prism-adaptation for some Ss.

\section{CONCLUSIONS}

\section{The Importance of Target-Pointing}

It may be concluded that the experience of relating the body to a stable visual target has an enhancing effect on visuomotor adaptation as measured by NA (Experiments 1 and 2) and PS (Experiment 2), and that this enhancement continues over a relatively prolonged prism-exposure period (Experiment 2). The finding of a small but significant degree of adaptation for the targetless conditions of both experiments probably indicates that target-pointing experience, while facilitative, is not absolutely necessary for visuomotor adaptation. It could be argued, however, that it is virtually impossible to arrange a situation in which $S$ is receiving no experience whatsoever in relating his visually displaced body to a stable visual object. For example, even in a situation such as that used by Held and his associates (Held \& Gottlieb, 1958 ), in which $S$ views his arm as it moves from side to side in front of a homogeneous background, there is the possibility that he is relating the image of the arm to the border of his visual field. It may be, therefore, that the difference between so-called targetless and target-pointing conditions is always a matter of degree.

\section{An Explanation of the Major Result}

The question of interest, of course, is why pointing at targets enhances visuomotor adaptation. The "Information Hypothesis" holds that many different sources of information serve to elicit adaptation and that the greater the sum of relevant information, the more complete the adaptation. According to this notion, the enhancement of adaptation for target-pointing $S$ s in the present experiments resulted from the added information that they received when making errors in localization. It must be pointed out, however, that target-pointing Ss were not only receiving an increase in information concerning the nature of the visual rearrangement, but were also being allowed to practice the motor response necessary to compensate for it. If one views motoric adaptation as an altered visuomotor response that is not consciously perceived as a correction (hence, its continuation after the distortion is removed), it is reasonable to assume that practicing the response would speed up its "normalization," resulting in a large negative aftereffect ${ }^{4}$ In the present experiments target-pointing error information and practice of the corrective response were confounded and, thus, further studies will be necessary to distinguish their respective effects.

\section{The Possibility of "Dual-Adaptation"}

A consistent finding of the two experiments was that NA significantly exceeded PS. It has already been indicated that the difference in size was probably not a methodological

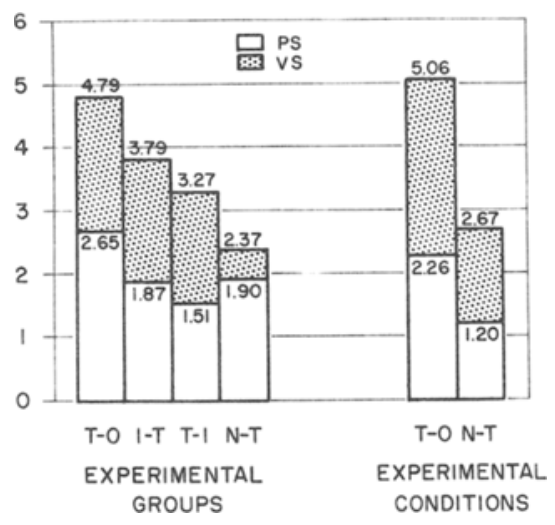

Fig. 2. Division of mean $(\mathrm{cm})$ negative aftereffect into proprioceptive (PS) and visual (VS) shifts for Experiments 1 and 2.

artifact, since in neither experiment were the two measures correlated with each other. A possible alternative explanation is that two types of adaptation took place - one proprioceptive and the other in some manner involving the visual system. If "dual adaptation" occurred, then NA was larger than PS because when the former was measured the corrective shifts in both modalities were revealed, but with eyes shut and felt finger position being measured only the shift in proprioception was recorded. Fig. 2 depicts the subdivision of NA into its proposed proprioceptive (PS) and visual (VS) shifts for both experiments. Evidence that suggests the existence of more than one component of the adapted state has been provided by a number of investigators (e.g., Hay \& Pick, 1966; Kalil \& Freedman, 1966; Cohen, 1967; McLaughlin \& Webster, 1967). Further research will be necessary, however, before much credence can be given this dual-component theory of adaptation as it applies to the present experimental situation.

\section{The Effect of Target-Pointing on PS}

One final question concerns the finding in Experiment 2 of a significant difference between the T-O and N-T conditions in regard to PS. According to Harris, $S$ must merely see his actively moved and visually displaced limb in order for the presumably automatic PS to occur. Furthermore, the greater the shift in felt body position, the greater the change in pointing behavior. However, the present results (at least those of Experiment 2) appear to reverse this cause-effect relation. That is, being forced to make the correct pointing response appears to lead to a greater PS than is the case if $S$ is in a situation that does not require immediate and deliberate compensation for visual displacement. Rock (1966) suggests that shift in felt body position is the result of S's coming to imagine the location of his body to be where he has seen it during the adaptation period. Rock's hypothesis suggests a possible explanation of the finding of Experiment 2 in regard to PS. It may be that when S is allowed to point at a target and is thus forced to compensate for the visual rearrangement, the rapidly acquired motor correction is accompanied by a ready acceptance of the visual image of the hand as indicative of its "true" position. This suggestion is plausible, since the motor correction is also rapidly coming to be perceived as normal. In a situation in which no target is present $S$ is not forced to immediately adjust to the visual displacement and, thus, the image of the hand is not as quickly or completely accepted as "true." Hence, when an $S$ who has received targetless prism-exposure estimates the location of his prism-exposed finger with his eyes shut, he does not imagine it 
to be as far to the side of the visual displacement as does an $\mathrm{S}$ who has been allowed to point at a target during the adaptation period. It is not apparent why Experiment 1 failed to demonstrate the clear-cut relationship between PS and target-pointing experience that was seen in Experiment 2. Further investigation will be required in order to determine the conditions necessary to produce this relationship.

\section{REFERENCES}

COHEN, M. M. Continuous versus terminal visual feedback in prism aftereffects. Perceptual \& Motor Skills, 1967, 24, 1295-1302.

COREN, S. Adaptation to prismatic displacement as a function of available information. Psychonomic Science, 1966, 4, 407-408.

HARRIS, C. S. Adaptation to displaced vision: A proprioceptive change. (Doctoral dissertation, Harvard University) Ann Arbor, Mich. University Microfilms, 1963a, No. 63-8162.

HARRIS, C. S. Adaptation to displaced vision: Visual, motor, or proprioceptive change? Science, 1963b, 140,812-813.

HARRIS, C. S. Proprioceptive changes underlying adaptation to visual distortions. American Psychologist, 1964, 19, 562 (Abstract).

HARRIS, C. S. Perceptual adaptation to inverted, reversed, and displaced vision. Psy chological Review, 1965, 72, 419-444.

HAY, J. C., \& PICK, H. L, JR. Visual and proprioceptive adaptation to optical displacement of the visual stimulus. Journal of Experimental Psychology, 1966, 71, 150-158.

HAYS, W. L. Statistics. New York: Holt, Rinehart, \& Winston, Inc., 1963.

HELD, R. Exposure-history as a factor in maintaining stability of perception and coordination. Journal of Nervous \& Mental Disease, $1961,132,26-32$.

HELD, R. Adaptation to rearrangement and visual-spatial aftereffects. Psychologische Beitrage, 1962, 6, 439-450.

HELD, $R$. The role of movement in the origin and maintenance of visual perception. Acta Psychologica, 1964, 23, 308-309.

HELD, R., \& BOSSOM, J. Neonatal deprivation and adult rearrangement: Complementary techniques for analyzing plastic sensory-motor coordinations. Journal of Comparative \& Physiological Psychology, $1961,54,33-37$

HELD, R., \& FREEDMAN, S. J. Plasticity in human sensorimotor control. Science, 1963, 142, 455-462.

HELD, R., \& GOTTLIEB, N. A technique for studying adaptation to disarranged handeye coordination. Perceptual \& Motor Skills, 1958 , $8,83-86$.
HELD, R., \& HEIN, A. Adaptation to disarranged hand-eye coordination contingent upon re-afferent stimulation. Perceptual \& Motor Skills, $1958,8,87-90$.

HELD, R., \& MIKAELIAN, H. Motor-sensory feedback versus need in adaptation to rearrangement. Perceptual \& Motor Skills, 1964, 18, 685-688.

HELD, R., \& SCHLANK, M. Adaptation to disarranged eye-hand coordination in the distance-dimension. American Journal of Psy chology, 1959, 72, 603-605

HOWARD, I. P., \& TEMPLETON, W. B. Humen spatial orientation. New York: John Wiley and Sons, 1966.

KALIL, R. E., \& FREEDMAN, S. J. Intermanual transfer of compensation for displaced vision. Perceptual \& Motor Skills, 1966, $22,123-126$.

MCLAUGHLIN, S. C., \& WEBSTER, R. G. Changes in straight-ahead eye position during adaptation to wedge prisms. Perception \& Psychophysics, 1967, 2, 37-44.

ROCK, I. The nature of perceptual adaptation. New York: Basic Books, 1966.

WALLACH, H. Informational discrepancy as a basis of perceptual adaptation. In S. J. Freedman (Ed.), The neuropsychology of spatially oriented behavior. Homewood, Ill.: The Dorsey Press, 1968. Pp. 209-229.

WALLACH, H., \& KARSH, E. B. The modification of stereoscopic depth-perception and the kinetic deptheffect. American Journal of Psychology, 1963, 76, 429-435.

\section{NOTES}

1. The experiments described formed the basis of a doctoral dissertation that was completed at the University of Oregon, June, 1967. The author wishes to thank Drs. Fred Attneave, Jacob Beck, and Robert Leeper, whose suggestions and criticisms aided immeasurably in the successful completion of this dissertation.

2. Address: Department of Psychology, University of Kansas, Lawrence, Kansas 66044

3. Howard and Templeton (1966) report making a more casual observation of the same phenomenon.

4. This notion was originally proposed by Howard and Templeton (1966).

(Accepted for publication November 18, 1968.) 